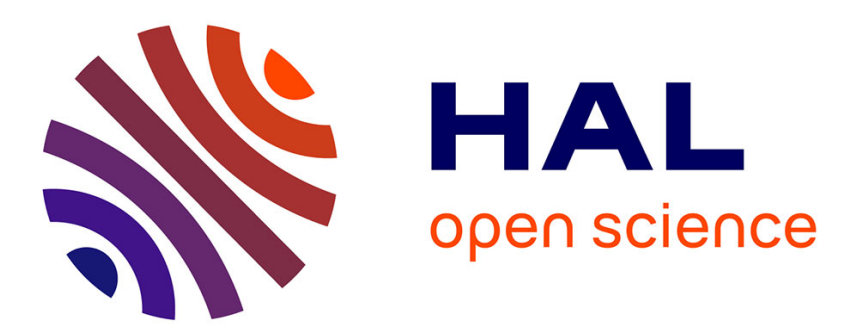

\title{
Evaluation des bornes de l'état d'un système incertain. Approche par intervalles
}

\author{
Estelle Cherrier, Mohamed Boutayeb, José Ragot
}

\section{To cite this version:}

Estelle Cherrier, Mohamed Boutayeb, José Ragot. Evaluation des bornes de l'état d'un système incertain. Approche par intervalles. Journal Européen des Systèmes Automatisés (JESA), 2003, 37, pp.1181. hal-00118523

\section{HAL Id: hal-00118523 https://hal.science/hal-00118523}

Submitted on 5 Dec 2006

HAL is a multi-disciplinary open access archive for the deposit and dissemination of scientific research documents, whether they are published or not. The documents may come from teaching and research institutions in France or abroad, or from public or private research centers.
L'archive ouverte pluridisciplinaire HAL, est destinée au dépôt et à la diffusion de documents scientifiques de niveau recherche, publiés ou non, émanant des établissements d'enseignement et de recherche français ou étrangers, des laboratoires publics ou privés. 


\title{
Evaluation des bornes de l'état d'un système incertain
}

\section{Approche par intervalles}

\author{
Estelle Cherrier**** - Mohamed Boutayeb** - José Ragot* \\ * CRAN UMR CNRS 7039 \\ INPL 2, Avenue de la Forêt de Haye \\ F-54516 Vandoeuvre-lès-Nancy Cedex \\ cherrier@eavr.u-strasbg.fr, jragot@ensem.inpl-nancy.fr \\ ** LSIIT UMR CNRS 7005 \\ ULP, Pôle API, Bd Sébastien Brandt \\ $B P 10413$ \\ F-67412 Illkirch \\ mohamed.boutayeb@ipst-ulp.u-strasbg.fr
}

RÉSUMÉ. Dans cet article, nous proposons une nouvelle approche formelle pour l'évaluation des bornes de l'état d'un système incertain. La méthode qui en résulte utilise une représentation par intervalles, et repose sur la construction d'un système dynamique augmenté, où le nouveau vecteur d'état est composé des bornes inférieure et supérieure de l'état du système incertain. Il est important de noter que nous considérons des incertitudes structurées, où seules les bornes de chaque coefficient des matrices du système sont supposées connues.

ABSTRACT. In this note we propose a new and formal approach for state bounds evaluation of uncertain systems. The method is based on the interval approach that leads to an augmented dynamical system where the new state vector is composed of the upper and lower bounds of the state. One of the main features is that we consider structural uncertainties where only bounds of each component of the state matrix are assumed to be known.

MOTS-CLÉS : systèmes incertains, enveloppe, intervalle, simulation, stabilité.

KEYWORDS: Uncertain Systems, Envelope, Interval, Simulation, Stability.

RS - JESA - Volume 37/2003. Outils de calculs ensemblistes, pages 1181 à 1192 


\section{Introduction}

Les deux dernières décennies ont été marquées par une intense activité de recherche sur l'analyse d'estimateurs et de contrôleurs robustes pour les systèmes incertains. Cependant, peu de résultats ont été élaborés pour l'analyse de ces systèmes. Les paramètres incertains apparaissent naturellement lors de la phase de modélisation : imprécision des capteurs, erreurs de modèles, incertitudes paramétriques ... et contribuent à définir l'incertitude globale des systèmes.

D'une manière générale, on distingue deux approches pour la synthèse des systèmes incertains. La première consiste à considérer les paramètres comme des réalisations de processus aléatoires. La seconde prend seulement en compte les bornes des intervalles caractérisant chaque incertitude (pour plus de détails concernant la détermination de ces bornes, voir [MUR 01]). Dans cette étude, nous nous intéresserons à l'évaluation des bornes de l'état par la seconde approche, car les densités de probabilité, utilisées dans la première méthode, ne sont pas toujours disponibles. Diverses méthodes ont été décrites pour simuler les systèmes incertains et générer une enveloppe contenant les variables d'état. La simulation d'un système dynamique à paramètres incertains appartenant à un intervalle conduit naturellement à la notion d'inclusion différentielle [RAC 96]. La résolution de l'inclusion différentielle correspondant au système incertain à simuler aboutit à la solution exacte, c'est-à-dire à « l'espace atteignable », donc aux enveloppes exactes contenant toutes les trajectoires possibles de chaque variable d'état, et uniquement celles-là. Cependant, la théorie des inclusions différentielles donne beaucoup de résultats fondamentaux, qui se révèlent difficiles à appliquer en pratique. C'est pourquoi dans [RAC 96], l'auteur tente de déterminer les bornes de cet espace atteignable en utilisant la notion de « quasi-trajectoire » de l'état, dont l'ensemble, sous certaines conditions de régularité du système incertain, est confondu avec l'espace atteignable. Cette méthode utilise des techniques de tirs aléatoires de trajectoires, dont la répartition doit converger vers une répartition uniforme au sein de l'enveloppe exacte. Elle n'est donc pas fondée sur une modélisation stochastique des systèmes incertains, mais fait appel à des outils probabilistes pour déterminer les bornes de l'enveloppe exacte. Il s'agit donc d'une méthode qui converge vers la véritable enveloppe, mais il n'est pas question dans cet article de mesurer l'erreur entre l'enveloppe générée et l'enveloppe exacte.

En comparant les performances de méthodes quantitatives, qualitatives, et semiqualitatives pour simuler des systèmes incertains dans [ARM 99], les auteurs développent la notion d'erreur entre une enveloppe obtenue par simulation et l'enveloppe exacte; ils proposent également une méthode pour obtenir des enveloppes dont l'erreur par rapport à l'enveloppe exacte est bornée, voire ajustable, grâce à la théorie des intervalles modaux. En effet, les simulations quantitatives ne peuvent donner qu'une sous-estimation de l'enveloppe exacte, tandis que les techniques qualitatives, purement numériques, connaissent des problèmes liés à la précision de l'implantation, et nécessitent des coûts de calculs importants. Enfin, les méthodes semi-qualitatives, c'est-à-dire fondées sur l'arithmétique des intervalles, mènent à des surestimations (comme nous le verrons par la suite), car elles ont recours à des techniques d'opti- 
misation globale de fonctions non linéaires et non convexes de plusieurs variables. [ARM 99] propose une amélioration de cette dernière méthode semi-qualitative, en mesurant le « degré de sur-estimation » de l'enveloppe générée, grâce à la sémantique des intervalles modaux : les auteurs donnent un algorithme pour construire une sous-enveloppe et une sur-enveloppe, et ensuite mesurer puis gérer la distance maximale entre ces deux enveloppes qui bornent l'enveloppe réelle. Il s'agit donc d'une approximation, ou plus exactement d'un encadrement de l'enveloppe exacte.

L'arithmétique floue peut être utilisée pour pallier l'effet d'enveloppe (ou effet enveloppant) qui se produit lors de la simulation d'un système incertain par des techniques fondées sur l'arithmétique «classique » des intervalles [HAN 02]. Une méthode dite de «transformation » gère les intervalles selon les principes de l'arithmétique floue. Les calculs d'optimisation ne sont pas menés directement sur les bornes des intervalles, mais sur des tableaux et des vecteurs obtenus en regroupant de différentes manières les bornes des intervalles contenant les paramètres du système incertain. Cela conduit à faire des évaluations multiples des fonctions à optimiser, pour obtenir des hypercubes qui sont soumis de façon limitée à des effets d'enveloppe. Le principal inconvénient de cette méthode réside dans son coût d'implantation élevé.

Une autre approche [ELG 99] est basée sur la représentation fractionnelle linéaire des systèmes incertains (c'est-à-dire la généralisation au cas multivariable et incertain de la représentation d'état d'un système par sa fonction de transfert). Cette méthode utilise la programmation semi-définie pour générer des « ellipsoïdes de confiance » de taille minimale, c'est-à-dire trouver un ellipsoïde contenant le vecteur d'état à un intant $\mathrm{k}+1$, connaissant l'ellipsoïde contenant le vecteur d'état à un intant $\mathrm{k}$. Le problème principal reste la surestimation des ellipsoïdes (il est difficile de garantir que l'ellipsoïde minimal trouvé à chaque étape correspond à l'ellipsoïde réel), et par ailleurs, cette méthode ne peut s'appliquer que sur un horizon temporel de taille finie.

Ici, nous nous intéressons aux méthodes fondées sur l'arithmétique des intervalles [MOO 79]. Un des écueils de ces méthodes est l'apparition d'un « effet d'enveloppe » (voir [JAU 01]), impossible à prévoir, qui rend difficilement exploitables les enveloppes générées : cet effet se produit lorsqu'il y a des variables à occurences multiples dans le modèle du système, ou lorsqu'il y a des dépendances entre les paramètres du système.

Pour prévenir cet effet d'enveloppe, nous proposons une nouvelle méthode pour l'évaluation des bornes de l'état d'un système incertain, utilisant la représentation des intervalles par leur centre et leur rayon. Nous obtenons ainsi un système dynamique augmenté, dont la stabilité est liée à l'effet d'enveloppe qui peut altérer l'enveloppe du système incertain initial.

Ce papier est organisé comme suit. Dans la section 2, nous définissons un système dynamique augmenté à partir d'un système incertain quelconque, et nous étudions sa stabilité en donnant une conditon suffisante, méthode qui sera appliquée sur un exemple numérique à la section 3 . 
Dans cet article, nous étudions les systèmes linéaires discrets à paramètres incertains. Nous nous intéresserons aux systèmes incertains de la forme :

$$
x_{k+1}=A_{k} x_{k}+B_{k} u_{k}, A_{k}=A+\Delta_{k}
$$

où $x$ et $u$ représentent respectivement l'état et la commande du système et où $\Delta_{k}$ représente la matrice des incertitudes paramétriques. Cela signifie que, à un instant donné $k$, les valeurs réelles des coefficients de la matrice d'état $A_{k}$ ne sont pas connues. La seule information disponible réside dans la connaissance des bornes des intervalles auxquels appartiennent ces coefficients.

\section{Systèmes générateurs d'enveloppes}

\subsection{Systèmes du premier ordre}

Dans un premier temps, on considère le cas scalaire autonome pour obtenir les formules analytiques des bornes de l'enveloppe du système : $x_{k+1}=\left(a+\delta_{k}\right) x_{k}$, où $\left|\delta_{k}\right| \leq \delta, \delta>0$ connu et les bornes $\underline{a}:=a-\delta$ et $\bar{a}:=a+\delta$ sont supposées de même signe (cette hypothèse est utilisée uniquement pour alléger la présentation, elle peut être aisément occultée).

Pour expliciter les bornes $\underline{x}$ et $\bar{x}$ de $x$, il faut distinguer deux cas selon le signe (fonction notée sgn) commun de $\underline{a}$ et $\bar{a}$ (pour alléger les formules, on note $\underline{s g n_{k}}=$ $\operatorname{sgn}\left(\underline{x}_{k}\right)$ et $\left.\overline{\operatorname{sgn}}_{k}=\operatorname{sgn}\left(\bar{x}_{k}\right)\right)$ :

$$
\begin{array}{r}
a>0 \Rightarrow\left\{\begin{aligned}
\underline{x}_{k+1} & =a \underline{x}_{k}-\delta\left|\underline{x}_{k}\right| \\
& =\underline{x}_{k}\left(a-\delta s g n_{k}\right) \\
\bar{x}_{k+1} & =a \bar{x}_{k}+\delta\left|\bar{x}_{k}\right| \\
& =\bar{x}_{k}\left(a+\delta \overline{s g n}_{k}\right)
\end{aligned}\right. \\
a<0 \Rightarrow\left\{\begin{aligned}
\underline{x}_{k+1} & =a \bar{x}_{k}-\delta\left|\bar{x}_{k}\right| \\
& =\bar{x}_{k}\left(a-\delta \overline{s g n}_{k}\right) \\
\bar{x}_{k+1} & =a \underline{x}_{k}+\delta\left|\underline{x}_{k}\right| \\
& =\underline{x}_{k}\left(a+\delta \underline{s g n}_{k}\right)
\end{aligned}\right.
\end{array}
$$

On regroupe les résultats (2) et (3) sous la forme :

$$
\left\{\begin{array}{l}
\underline{x}_{k+1}=\frac{1}{2}\left[\underline{x}_{k}\left(a-\delta \underline{s g n}_{k}\right)(1+\operatorname{sgn}(a))+\bar{x}_{k}\left(a-\delta \overline{s g n}_{k}\right)(1-\operatorname{sgn}(a))\right] \\
\bar{x}_{k+1}=\frac{1}{2}\left[\bar{x}_{k}\left(a+\delta \overline{s g n}_{k}\right)(1+\operatorname{sgn}(a))+\underline{x}_{k}\left(a+\delta \underline{s g n}_{k}\right)(1-\operatorname{sgn}(a))\right]
\end{array}\right.
$$

Les caractéristiques (centre $m_{k}$ et rayon $\delta_{k}$ ) de l'intervalle $\left[x_{k}\right]$ contenant l'état à l'instant $\mathrm{k}$ sont données par :

$$
\left\{\begin{aligned}
m_{k} & =\frac{1}{2}\left(\bar{x}_{k}+\underline{x}_{k}\right) \\
\delta_{k} & =\frac{1}{2}\left(\bar{x}_{k}-\underline{x}_{k}\right)
\end{aligned}\right.
$$


Finalement, en reportant (4) dans (5), on aboutit, à l'instant k+1, à l'expression :

$$
\left\{\begin{array}{c}
m_{k+1}=\frac{1}{2}\left(a\left(\underline{x}_{k}+\bar{x}_{k}\right)+\delta \operatorname{sgn}(a)\left(\left|\underline{x}_{k}\right|+\left|\bar{x}_{k}\right|\right)\right) \\
\delta_{k+1}=\frac{1}{2}\left(|a|\left(\bar{x}_{k}-\underline{x}_{k}\right)+\delta\left(\left|\underline{x}_{k}\right|+\left|\bar{x}_{k}\right|\right)\right)
\end{array}\right.
$$

De façon condensée, on obtient :

$$
\left[\begin{array}{c}
m_{k+1} \\
\delta_{k+1}
\end{array}\right]=\frac{1}{2}\left[\begin{array}{cc}
a & a \\
-|a| & |a|
\end{array}\right]\left[\begin{array}{l}
\underline{x}_{k} \\
\bar{x}_{k}
\end{array}\right]+\frac{1}{2}\left[\begin{array}{cc}
-\delta \operatorname{sgn}(a) & \delta \operatorname{sgn}(a) \\
\delta & \delta
\end{array}\right]\left[\begin{array}{c}
\left|\underline{x}_{k}\right| \\
\left|\bar{x}_{k}\right|
\end{array}\right]
$$

On démontrera à la section suivante, dans le cas général d'un système d'ordre $n \geq 1$, la validité de ces formules.

\subsection{Systèmes d'ordre n}

Dans cette section, on généralise les formules (7) au cas matriciel, en supposant que les variables sont indépendantes, c'est-à-dire que l'on ne prend pas en compte les liens qui peuvent exister entre les coefficients de la matrice d'état $A_{k}$ du système.

On considère le système incertain $x_{k+1}=A_{k} x_{k}+B u_{k}$, dont la matrice d'état est incertaine, i.e. de la forme $A_{k}=A+\Delta_{k}$, où $\Delta_{k}=\left\{\delta_{i j}(k)\right\}_{i, j=1, n},\left|\delta_{i j}(k)\right| \leq$ $\delta_{i j}$, et $\Delta=\left\{\delta_{i j}\right\}_{i, j=1, n}$. La matrice intervalle $\Delta_{k}$ est donc incertaine, au sens où la seule information disponible réside dans les bornes des intervalles dans lesquels varient ses coefficients. On rappelle l'hypothèse faite, selon laquelle le signe de tous les coefficients intervalles ne change pas au cours du temps (les bornes des intervalles définissant les matrices du système sont de même signe).

Par ailleurs, on peut remarquer [MOO 79] qu'un vecteur intervalle $[x]$ est caractérisé soit par son centre $m_{x}$ et son rayon $\delta_{x}$, soit par ses bornes inférieure et supérieure $\underline{x}$ et $\bar{x}$, ces deux représentations étant liées par la transformation :

$$
\left[\begin{array}{c}
\underline{x}_{k} \\
\bar{x}_{k}
\end{array}\right]=\left[\begin{array}{cc}
I_{n} & -I_{n} \\
I_{n} & I_{n}
\end{array}\right]\left[\begin{array}{c}
m_{x} \\
\delta_{x}
\end{array}\right]
$$

où $I_{n}$ est la matrice identité de rang $\mathrm{n}$.

L'expression (7) se généralise (pour un système autonome) en remplaçant $a$ par A et $\delta$ par $\Delta$ :

$$
\left[\begin{array}{c}
m_{k+1} \\
\delta_{k+1}
\end{array}\right]=\frac{1}{2}\left[\begin{array}{cc}
A & A \\
-|A| & |A|
\end{array}\right]\left[\begin{array}{c}
\underline{x}_{k} \\
\bar{x}_{k}
\end{array}\right]+\frac{1}{2}\left[\begin{array}{cc}
-\Delta * S_{A} & \Delta * S_{A} \\
\Delta & \Delta
\end{array}\right]\left[\begin{array}{c}
\left|\underline{x}_{k}\right| \\
\left|\bar{x}_{k}\right|
\end{array}\right]
$$

où $*$ est le produit de Hadamard, la matrice $S_{A}=\operatorname{sgn}(A)$ (la définition de la fonction sgn est étendue naturellement aux matrices réelles), et $|A|$ désigne la matrice dont les coefficients sont les valeurs absolues des coefficients correspondants de $A$. Il vient, d'après (8), (9), pour un système muni d'une commande $u$ :

$$
\left[\begin{array}{l}
\underline{x}_{k+1} \\
\bar{x}_{k+1}
\end{array}\right]=M\left[\begin{array}{l}
\underline{x}_{k} \\
\bar{x}_{k}
\end{array}\right]+N\left[\begin{array}{l}
\left|\underline{x}_{k}\right| \\
\left|\bar{x}_{k}\right|
\end{array}\right]+E u_{k}
$$


avec

$$
\begin{aligned}
& M=\frac{1}{2}\left[\begin{array}{ll}
A+|A| & A-|A| \\
A-|A| & A+|A|
\end{array}\right] \\
& N=\frac{1}{2}\left[\begin{array}{cc}
-\Delta-\Delta * S_{A} & -\Delta+\Delta * S_{A} \\
\Delta-\Delta * S_{A} & \Delta+\Delta * S_{A}
\end{array}\right] \\
& E=\left[\begin{array}{l}
B \\
B
\end{array}\right]
\end{aligned}
$$

On pose alors $z_{k}=\left[\underline{x}_{k} \bar{x}_{k}\right]^{T}$, et on obtient finalement le système d'ordre $2 n$ :

$$
z_{k+1}=M z_{k}+N\left|z_{k}\right|+E u_{k}
$$

Ainsi $z_{k}$ peut être considéré comme un vecteur enveloppe du système incertain, car il est constitué de la borne inférieure et de la borne supérieure de ce système. Le système défini par (12) est ainsi appelé « système générateur d'enveloppe ».

Pour terminer cette partie, on va prouver la validité de l'enveloppe, c'est-à-dire la véracité des formules (9) et (10). On montre par récurrence sur k la propriété $\left(H_{k}\right)$ :

$$
\underline{x}_{k} \leq x_{k} \leq \bar{x}_{k}
$$

Par définition d'un intervalle, on a immédiatement $x_{0} \in\left[\underline{x}_{0}, \bar{x}_{0}\right]$, donc la propriété est vraie au rang 0 .

Soit alors k positif tel que $\left(H_{k}\right)$ est vraie. On montre que sous cette hypothèse, la propriété $\underline{x}_{k} \leq x_{k}$ est vraie au rang $\mathrm{k}+1$ ('’autre inégalité de la propriété (13) se démontre de façon analogue).

On sait, d'après la définition du problème, que :

$$
x_{k+1}=\left(A+\Delta_{k}\right) x_{k}+B u_{k}
$$

D'autre part, en développant la formule (10), on obtient :

$\underline{x}_{k+1}=\frac{1}{2}(A+|A|) \underline{x}_{k}+\frac{1}{2}(A-|A|) \bar{x}_{k}-\frac{1}{2}\left(\Delta+\Delta * S_{A}\right)\left|\underline{x}_{k}\right|+\frac{1}{2}\left(-\Delta+\Delta * S_{A}\right)\left|\bar{x}_{k}\right|+B u_{k}$

D'où par soustraction de (14) et (15) :

$$
\begin{aligned}
x_{k+1}-\underline{x}_{k+1}= & A\left(x_{k}-m_{k}\right)+\Delta_{k} x_{k}+|A| \delta_{k} \\
& +\frac{1}{2} \Delta\left(\left|\underline{x}_{k}\right|+\left|\bar{x}_{k}\right|\right)+\frac{1}{2} \Delta * S_{A}\left(\left|\underline{x}_{k}\right|-\left|\bar{x}_{k}\right|\right)
\end{aligned}
$$

On étudie ensuite le signe de chaque composante i de ce vecteur différence $(i \in[1, n]$ et on note $a_{i j}$ les coefficients de la matrice A, etc $\left.\ldots, i, j \in[1, n]\right)$ :

$$
\begin{aligned}
x_{k+1}(i)-\underline{x}_{k+1}(i) & =\sum_{j=1}^{n}\left[a_{i j}\left(x_{k}(j)-m_{k}(j)\right)+\Delta_{k}(i j) x_{k}(j)+\left|a_{i j}\right| \delta_{k}(j)\right. \\
& \left.+\Delta_{i j}\left(\frac{\left|\underline{x}_{k}(j)\right|}{2}+\frac{\left|\bar{x}_{k}(j)\right|}{2}+\operatorname{sgn}\left(a_{i j}\right)\left(\frac{\left|\underline{x}_{k}(j)\right|}{2}-\frac{\left|\bar{x}_{k}(j)\right|}{2}\right)\right)\right]
\end{aligned}
$$


On note $\Sigma_{j}$ chaque terme de la somme précédente. On fixe alors $j \in[1, n]$ :

$$
\begin{aligned}
- \text { Si } a_{i j} & \geq 0: \\
\Sigma_{j} & =a_{i j}\left(x_{k}(j)-m_{k}(j)+\delta_{k}(j)\right)+\Delta_{k}(i j) x_{k}(j)+\Delta_{i j}\left|\underline{x}_{k}(j)\right|
\end{aligned}
$$

Compte tenu de la définition (5), on a aussi :

$$
\begin{aligned}
\Sigma_{j} & =a_{i j}\left(x_{k}(j)-\underline{x}_{k}(j)\right)+\Delta_{k}(i j) x_{k}(j)+\Delta_{i j}\left|\underline{x}_{k}(j)\right| \\
& =\left(a_{i j}+\Delta_{k}(i j)\right) x_{k}(j)-a_{i j} \underline{x}_{k}(j)+\Delta_{i j}\left|\underline{x}_{k}(j)\right| \\
& =\left(a_{i j}+\Delta_{k}(i j)\right) x_{k}(j)+\left(-a_{i j}+\Delta_{i j} \operatorname{sgn}\left(\underline{x}_{k}(j)\right) \underline{x}_{k}(j)\right.
\end{aligned}
$$

Or $\underline{x}_{k}(j)$ est une constante, et par hypothèse sur le signe des coefficients de la matrice intervalle $A_{k}, a_{i j}+\Delta_{k}(i j) \geq 0, \forall k$. Donc $\Sigma_{j}$ est une fonction croissante de $x_{k}(j)$. En particulier :

$$
\begin{aligned}
\Sigma_{j} & \geq\left(a_{i j}+\Delta_{k}(i j)\right) \underline{x}_{k}(j)+\left(-a_{i j}+\Delta_{i j} \operatorname{sgn}\left(\underline{x}_{k}(j)\right)\right) \underline{x}_{k}(j) \\
& \geq\left(\Delta_{k}(i j)+\Delta_{i j} \operatorname{sgn}\left(\underline{x}_{k}(j)\right)\right) \underline{x}_{k}(j)
\end{aligned}
$$

On distingue alors deux cas :

$$
\begin{aligned}
& \text { - si } \underline{x}_{k}(j) \geq 0, \text { alors }\left(\Delta_{k}(i j)+\Delta_{i j} \operatorname{sgn}\left(\underline{x}_{k}(j)\right)\right) \geq 0 \\
& \text { - si } \underline{x}_{k}(j) \leq 0, \text { alors }\left(\Delta_{k}(i j)+\Delta_{i j} \operatorname{sgn}\left(\underline{x}_{k}(j)\right)\right) \leq 0
\end{aligned}
$$

Donc, dans tous les cas, $\Sigma_{j} \geq 0$.

- Si $a_{i j} \leq 0$ : un raisonnement analogue montre qu'on obtient le même résultat, à savoir que $\Sigma_{j} \geq 0$.

Finalement, d'après la formule (17), $x_{k+1}(i)-\underline{x}_{k+1}(i)$ est la somme de termes positifs, donc $x_{k+1}(i) \geq \underline{x}_{k+1}(i), \forall i \in[1, n]$, i.e. $x_{k+1} \geq \underline{x}_{k+1}$.

La propriété $\left(H_{k+1}\right)$ vient donc d'être démontrée, et le principe de récurrence assure que, pour tout $\mathrm{k}$ positif, $x_{k} \geq \underline{x}_{k}$. De même, $x_{k} \leq \bar{x}_{k}$

\subsection{Analyse de la stabilité}

\subsubsection{Etude théorique}

On cherche à caractériser la stabilité du système autonome associé à (12) (en utilisant la deuxième formulation de Lyapunov) :

$$
z_{k+1}=M z_{k}+N\left|z_{k}\right|
$$

où $z$ est le vecteur d'état obtenu en (12).

Tout d'abord, on considère l'inégalité standard :

$$
x^{T} A^{T} A x+y^{T} B^{T} B y \geq x^{T} A^{T} B y+y^{T} B^{T} A x
$$


obtenue en développant: $(A x-B y)^{T}(A x-B y) \geq 0$ (A et B sont des matrices de dimensions appropriées, $x$ et $y$ sont des vecteurs).

Si on remplace dans (22) $A$ par $S^{1 / 2} A$ et $B$ par $S^{-1 / 2} B$ (où $S$ est une matrice définie positive), on obtient :

$$
x^{T} A^{T} S A x+y^{T} B^{T} S^{-1} B y \geq x^{T} A^{T} B y+y^{T} B^{T} A x
$$

Introduisons la fonction de Lyapunov :

$$
V_{k}=z_{k}^{T} P z_{k}
$$

On calcule la variation entre deux instants :

$$
\Delta V_{k}=V_{k+1}-V_{k}=z_{k+1}^{T} P z_{k+1}-z_{k}^{T} P z_{k}
$$

On remplace alors $z_{k+1}$ par son expression (21) :

$$
\Delta V_{k}=\left(M z_{k}+N\left|z_{k}\right|\right)^{T} P\left(M z_{k}+N\left|z_{k}\right|\right)-z_{k}^{T} P z_{k}
$$

On développe :

$$
\Delta V_{k}=z_{k}^{T}\left(M^{T} P M-P\right) z_{k}+z_{k}^{T} M^{T} P N\left|z_{k}\right|+\left|z_{k}^{T}\right| M^{T} P N z_{k}+\left|z_{k}^{T}\right| N^{T} P N\left|z_{k}\right|
$$

Or, d'après l'inégalité (23) :

$z_{k}^{T} M^{T} P N\left|z_{k}\right|+\left|z_{k}^{T}\right| M^{T} P N z_{k} \leq z_{k}^{T} M^{T} P^{1 / 2} S P^{1 / 2} M z_{k}+\left|z_{k}^{T}\right| N^{T} P^{1 / 2} S^{-1} P^{1 / 2} N\left|z_{k}\right|$

S étant une matrice définie positive arbitraire.

En regroupant (27) et (28), on arrive à :

$$
\Delta V_{k} \leq z_{k}^{T}\left(M^{T}\left(P+P^{1 / 2} S P^{1 / 2}\right) M-P\right) z_{k}+\left|z_{k}^{T}\right| N^{T}\left(P+P^{1 / 2} S^{-1} P^{1 / 2}\right) N\left|z_{k}\right|
$$

On suppose que $\mathrm{P}$ est choisie de telle sorte que :

$$
M^{T}\left(P+P^{1 / 2} S P^{1 / 2}\right) M-P=-Q^{T} Q
$$

où $Q$ est une matrice inversible, et $S$ est définie positive.

Si on prend (30) en compte, (29) se réduit à :

$$
\Delta V_{k} \leq-z_{k}^{T} Q^{T} Q z_{k}+\left|z_{k}^{T}\right| N^{T}\left(P+P^{1 / 2} S^{-1} P^{1 / 2}\right) N\left|z_{k}\right|
$$

On note $w_{k}:=Q z_{k}$, et $\epsilon$ le coefficient de Lipschitz de la fonction : $z \mapsto N|z|$. On obtient :

$$
\begin{aligned}
\Delta V_{k} & \leq-w_{k}^{T} w_{k}+\left|Q^{-1} w_{k}^{T}\right| N^{T}\left(P+P^{1 / 2} S^{-1} P^{1 / 2}\right) N\left|Q^{-1} w_{k}\right| \\
& \leq\left(-1+\epsilon^{2}\left\|P+P^{1 / 2} S^{-1} P^{1 / 2}\right\|_{2}^{2}\left\|Q^{-1}\right\|_{2}^{2}\right)\left\|w_{k}\right\|_{2}^{2}
\end{aligned}
$$


Pour évaluer le coefficient de Lipschitz de la fonction : $z \mapsto N|z|$, remarquons que :

$$
\|N|z|\|_{2}^{2} \leq\|N\|_{F}^{2}\|z\|_{2}^{2}
$$

où $\|\cdot\|_{F}$ est la norme de Frobënius. On a donc $\epsilon \leq\|N\|_{F}$. En reportant dans (32), une condition suffisante pour que $\mathrm{V}$ soit une fonction de Lyapunov décroissante est donnée par :

$$
-1+\|N\|_{F}^{2}\left\|P+P^{1 / 2} S^{-1} P^{1 / 2}\right\|_{2}^{2}\left\|Q^{-1}\right\|_{2}^{2}<0
$$

On obtient finalement le théorème :

Théorème 2.1. S'il existe des matrices $P$ définie positive et $Q, S$ inversibles, telles que :

$$
\left\{\begin{array}{l}
M^{T}\left(P+P^{1 / 2} S P^{1 / 2}\right) M-P=-Q^{T} Q \\
-1+\|N\|_{F}^{2}\left\|P+P^{1 / 2} S^{-1} P^{1 / 2}\right\|_{2}^{2}\left\|Q^{-1}\right\|_{2}^{2}<0
\end{array}\right.
$$

alors le système (21) : $z_{k+1}=M z_{k}+N\left|z_{k}\right|$ est asymptotiquement stable.

En pratique, on peut rechercher une matrice $\mathrm{S}$ de la forme $S=\alpha I_{n}, \alpha>0$, et on obtient une condition plus simple :

$$
\left\{(1+\alpha) M^{T} P M-P=-Q^{T} Q,\|N\|_{F}^{2}<\frac{1}{(1+1 / \alpha)\|P\|_{2}^{2}\left\|Q^{-1}\right\|_{2}^{2}}\right.
$$

Ce théorème donne une condition suffisante pour garantir la stabilité des systèmes générateurs d'enveloppe. Lorsque les hypothèses du théorème 2.1 sont remplies, on peut garantir que l'enveloppe simulée contenant l'état du système incertain de départ ne sera pas soumise à un effet enveloppant.

\subsubsection{Algorithme}

Dans cette section, on détaille un algorithme qui permet de prédire si la simulation de l'enveloppe contenant l'état d'un système incertain est soumise à un effet enveloppant. Le but est de trouver, s'ils existent, un réel strictement positif $\alpha$, et deux matrices définies positives $P$ et $Q$ qui vérifient (35).

On suppose que l'on dispose d'un outil permettant de résoudre les équations matricielles du type $:(1+\alpha) M^{T} P M-P=-L$. On pose $L:=Q^{T} Q$, et on choisit arbitrairement $\alpha=\alpha_{0}>0$, et un pas d'incrémentation $p$.

Soit $\alpha \in \mathbb{R}_{+}^{*}$ fixé.

S'il existe une solution à l'équation :

$$
(1+\alpha) M^{T} P M-P=-L
$$

sous les conditions $P>0, L>0$, on détermine $Q$ telle que $L=Q^{T} Q$ (décomposition de Cholesky).

Dans ce cas, si la condition

$$
\|N\|_{F}^{2}<\frac{1}{(1+1 / \alpha)\|P\|_{2}^{2}\left\|Q^{-1}\right\|_{2}^{2}}
$$


est vérifiée, cela signifie que les hypothèses du théorème 2.1 sont remplies, l'enveloppe simulée ne subit pas d'effet enveloppant, on a bien trouvé $\alpha, P$ et $Q$ convenables.

Si l'une de ces conditions n'est pas remplie, l'algorithme s'arrête, la valeur testée pour le coefficient $\alpha$ ne convient pas, il faut tester une nouvelle valeur : $\alpha+p$.

Cette méthode est heuristique, et la condition vérifiée est une condition suffisante : cela signifie que si aucune valeur testée pour $\alpha$ ne permet de calculer des matrices $P$ et $Q$ convenables, on ne peut rien affirmer sur la qualité de l'enveloppe simulée, on ne pourra pas prédire s'il va se produire un effet d'enveloppe ou non.

\section{Exemple}

On cherche l'enveloppe contenant l'état du système à temps discret :

$$
x_{k+1}=A_{k} x_{k}+B u_{k}
$$

où $A_{k}=A+\Delta_{k}$ avec :

$$
A=\left[\begin{array}{cc}
0,85 & -0,1 \\
-0,1 & 0,75
\end{array}\right],\left|\Delta_{k}\right| \leq \Delta=\left[\begin{array}{cc}
0,01 & 0,005 \\
0,005 & 0,01
\end{array}\right]
$$

et

$$
B=\left[\begin{array}{cc}
0,4 & 0 \\
0,01 & 0,4
\end{array}\right]
$$

L'état initial $x_{0}$ est choisi égal à $\left[\begin{array}{ll}0 & 0\end{array}\right]^{T}$ et u est choisie pseudopériodique. Alors, les matrices du système générateur d'enveloppe correspondant sont données par :

$$
\begin{gathered}
M=\left[\begin{array}{cccc}
0,85 & 0 & 0 & -0,1 \\
0 & 0,75 & -0,1 & 0 \\
0 & -0,1 & 0,85 & 0 \\
-0,1 & 0 & 0 & 0,75
\end{array}\right] \\
N=\left[\begin{array}{cccc}
-0,01 & 0 & 0 & -0,005 \\
0 & -0,01 & -0,005 & 0 \\
0 & 0,005 & 0,01 & 0 \\
0,005 & 0 & 0 & 0,01
\end{array}\right]
\end{gathered}
$$

Si on prend $\alpha=0,1$, on peut trouver des matrices qui satisfont les conditions du théorème 2.1 :

$$
\begin{array}{r}
P=\left[\begin{array}{cccc}
1,3996 & 0 & 0 & 0,1336 \\
0 & 1,5332 & 0,1336 & 0 \\
0 & 0,1336 & 1,3996 & 0 \\
0,1336 & 0 & 0 & 1,5332
\end{array}\right] \\
Q=\left[\begin{array}{cccc}
0,5435 & 0 & 0 & 0,5442 \\
0 & 0,7689 & 0,3847 & 0 \\
0 & 0 & 0,3839 & 0 \\
0 & 0 & 0 & 0,5431
\end{array}\right]
\end{array}
$$


En simulant le système générateur (12), on constate sur cet exemple que les enveloppes contenant les deux variables d'état n'explosent pas (figure 1). Rappelons que l'ensemble des solutions de (36) est inclus dans le domaine délimité par ces deux enveloppes.
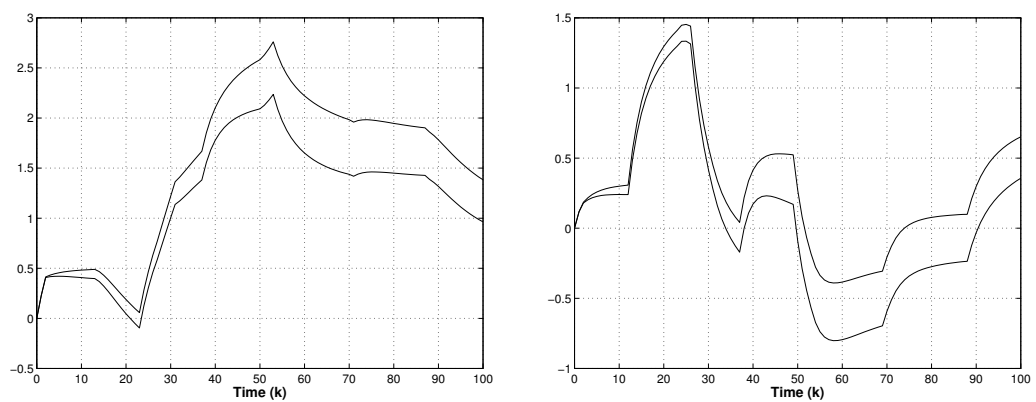

Figure 1. Enveloppes des variables d'état

\section{Conclusion}

Dans cet article, nous avons présenté une approche par intervalles pour évaluer les bornes du vecteur d'état d'un système linéaire incertain. Le point principal réside dans l'appréhension du problème sous la forme d'un système dynamique non linéaire augmenté (12). Nous avons alors pu analyser la stabilité de ce système par une technique de Lyapunov, et ainsi prévoir, grâce à une condition suffisante, l'effet d'enveloppe qui peut se produire lorsqu'on simule l'état d'un système incertain. L'efficacité de cette méthode heuristique a été testée sur un exemple numérique.

\section{Bibliographie}

[ARM 99] ARMEngol J., Application of Modal Interval Analysis to the simulation of the behaviour of dynamic systems with uncertain parameters, Thesis, Universitat de Girona, Catalonia, Spain, 1999.

[ELG 99] ElGhaoui L., CAlafiore G., « Worst-case State Prediction under Structured Uncertainty », Proceedings of the American Control Conference, San Diego, California, 26-28 June 1999, p. 3402-3406.

[HAN 02] HANSS M., « The transformation method for the simulation and analysis of systems with uncertain parameters », Fuzzy Sets and Systems, vol. 130, 2002, p. 277-289.

[JAU 01] JAUlin L., KiefFer M., Didrit O., Walter E., Applied Interval Analysis, Springer, 2001.

[MOO 79] MoORE R. E., Methods and Applications of Interval Analysis, Studies in Applied Mathematics, Philadelphia, Pennsylvania, 1979. 
1192 RS - JESA - Volume 37/2003. Outils de calculs ensemblistes

[MUR 01] Muramatsu E., IKeda M., « An Extended Parameter Estimation Method via Multiple Observers », Proceedings of the 40th IEEE Conference on Decision and Control, Orlando, Florida USA, 2001, p. 3314-3317.

[RAC 96] RACZYNSKI S., « Differential Inclusions in System Simulation », Transaction of the Society for Computer Simulation, vol. 13, $\mathrm{n}^{\circ}$ 1, 1996, p. 47-54. 